proteome of COPD sputum at exacerbation and stable disease. It suggests a role for MMP-12 in complement regulation and haemostasis in COPD. Thus an important peptide library has been unravelled, providing an ideal tool in developing drugs and understanding COPD pathogenesis.

\section{S130 AXL RECEPTOR TYROSINE KINASE ON AIRWAY MACROPHAGES HAS A KEY ROLE IN LUNG IMMUNE HOMEOSTASIS}

${ }^{1} \mathrm{~N}$ Denny, ${ }^{1} \mathrm{AM}$ Grabiec, ${ }^{2} \mathrm{G}$ Tavernier, ${ }^{2} \mathrm{~S}$ Holden, ${ }^{2} \mathrm{H}$ Francis, ${ }^{2} \mathrm{D}$ Ryan, ${ }^{2} \mathrm{R}$ Niven, ${ }^{2} \mathrm{SJ}$ Fowler, ${ }^{2} \mathrm{~A}$ Simpson, ${ }^{1} \mathrm{~T}$ Hussell. ${ }^{1}$ Manchester Collaborative Centre for Inflammation Research, The University of Manchester, Manchester, UK; ${ }^{2}$ Institute of Inflammation and Repair, Manchester Academic Health Sciences Centre, University of Manchester and National Institute of Health Research Respiratory and Allergy Clinical Research Facility, University Hospital of South Manchester, Manchester, UK

\subsection{6/thoraxjnl-2015-207770.136}

Rationale Apoptotic cell uptake (efferocytosis) by airway macrophages (AMs) is critical for lung immune homeostasis and is defective in chronic lung diseases, including asthma, ${ }^{1}$ although the molecular mechanism behind this remains unknown. The TAM (Tyro3, Axl, MerTK) receptor tyrosine kinases are one of the main receptor classes that mediate efferocytosis but little is known about their regulation and function in inflammatory lung diseases.

Aim To investigate expression profile of TAM receptors and their ligand Gas6 in human AMs and analyse potential defects in TAM receptor expression in chronic lung inflammation.

Methods AMs from the sputum of patients with asthma (BTS step 3-5) $(\mathrm{n}=30)$ or healthy donors (HD) $(\mathrm{n}=12)$ were enriched by plastic adhesion. Monocytes were isolated from matched whole blood samples by CD14 positive selection and differentiated into monocyte-derived macrophages (MDMs). Total RNA was extracted from all purified cell populations and mRNA expression analysed by qPCR. HD MDMs were stimulated with Th2 cytokines in vitro and TAM receptor expression was analysed using qPCR and ELISA.

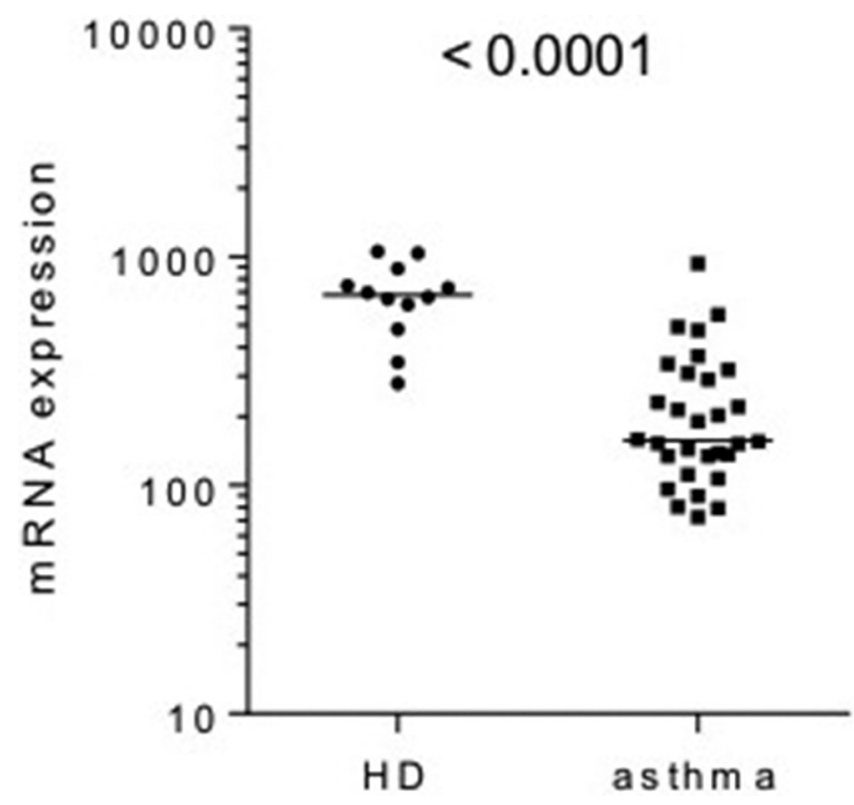

Abstract S130 Figure 1 mRNA expression of Axl in airway macrophages of healthy donors and patients with asthma
Main results Axl was the dominant TAM receptor expressed in HD AMs whereas monocytes and MDMs predominantly expressed MerTK. Axl expression was significantly reduced in AMs from patients with asthma compared to HD ( $\mathrm{p}<0.0001$ ), while mRNA levels of MerTK and Gas6 was similar in both groups. We found no differences in Axl and MerTK expression in monocytes and MDMs from HD and patients with asthma, indicating that the observed differences were restricted to the site of inflammation. In vitro, MDM stimulation with IL-4 or IL-13 downregulated Axl mRNA and protein expression in a time-dependent manner.

Conclusions We have shown for the first time that Axl is the principal TAM receptor expressed in human AMs. Significant reduction of Axl expression in AMs from patients with asthma might be responsible for inefficient clearance of apoptotic cells from the inflamed airways and contribute to persistent airway inflammation. Strategies aimed at restoration of Axl expression or activity may represent a novel therapeutic strategy in asthma and other chronic lung diseases.

\section{REFERENCE}

1 Simpson JL, Gibson PG, Yang IA et al. Impaired macrophage phagocytosis in non-eosinophilic asthma. Clin Exp Allergy. 2013;43:29-35

\section{S131 DEFICIENCY MUTATIONS OF $\alpha 1$-ANTITRYPSIN DIFFERENTIALLY AFFECT FOLDING, FUNCTION AND POLYMERISATION}

${ }^{1}$ I Haq, ${ }^{1} \mathrm{JA}$ Irving, ${ }^{2} \mathrm{AD}$ Saleh, ${ }^{3} \mathrm{~L}$ Dron, ${ }^{1} \mathrm{GL}$ Regan-Mochrie, ${ }^{1} \mathrm{~N}$ Motamedi-Shad, ${ }^{1} \mathrm{JR}$ Hurst, ${ }^{1}$ DA Lomas, ${ }^{4}$ B Gooptu. 'University College London, London, UK; ${ }^{2}$ Broomfield Hospital NHS, Chelmsford, UK; ${ }^{3}$ Royal Free Hospital, London, UK; ${ }^{4}$ King's College London, London, UK

\subsection{6/thoraxjnl-2015-207770.137}

Misfolding, polymerisation and defective secretion of functional $\alpha_{1}$-antitrypsin underlie the predispositions to severe liver and lung disease in $\alpha_{1}$-antitrypsin deficiency. We have identified a novel (Ala336Pro, Baghdad) deficiency variant and characterised it relative to the wild-type (M) and common severe $\mathrm{Z}$ (Glu342Lys) variant. The index case is a homozygous individual of consangineous parentage. Absolute levels of circulating $\alpha_{1}$ antitrypsin were in the moderate deficiency range but the biochemical phenotype could not be clearly classified by standard methods. Moreover the majority was polymerised, i.e. functionally inactive, and the purified monomer was only $37 \%$ active relative to the wild-type ' $\mathrm{M}$ ' variant. Together these resulted in 85$95 \%$ loss-of-function, a similarly severe functional deficiency to that of ZZ homozygotes. Biochemical, biophysical and computational studies further defined the molecular basis of this functional deficiency. These demonstrated that native Ala336Pro $\alpha_{1}$ antitrypsin could adopt the polymerogenic intermediate conformation and polymerised more readily not only than $\mathrm{M} \alpha_{1}$-antitrypsin but also the severe $\mathrm{Z}$ variant. Nevertheless folding was far less impaired in Ala336Pro $\alpha_{1}$-antitrypsin than in the $Z$ variant. The data therefore indicate partitions between the contribution of the 'breach' (site of $\mathrm{Z}$ mutation) and 'shutter' (Ala336Pro) regions of strand 5A to folding and to polymerisation mechanisms. Moreover the findings demonstrate that in these variants, folding efficiency does not correlate directly with the tendency to polymerise in vitro or in vivo. They therefore differentiate generalised misfolding from polymerisation tendencies in missense variants of $\alpha_{1}$-antitrypsin. Clinically they further support the need to quantify loss-of-function in $\alpha_{1}$-antitrypsin deficiency to individualise patient care. 


\section{Idiopathic pulmonary fibrosis boldly goes where no disease has gone before}

\section{P1 PRELIMINARY RESULTS FOR ASSOCIATION OF SURVIVAL TIME IN IDIOPATHIC PULMONARY FIBROSIS CASES WITH THE 11P15.5 REGION}

${ }^{1} \mathrm{RJ}$ Allen, ${ }^{1} \mathrm{MD}$ Tobin, ${ }^{1} \mathrm{LV}$ Wain, ${ }^{2} \mathrm{R}$ Braybrooke, ${ }^{2} \mathrm{G}$ Jenkins. ${ }^{1}$ Department of Health Sciences, University of Leicester, Leicester, UK; ${ }^{2}$ School of Medicine, University of Nottingham, Nottingham, UK

\subsection{6/thoraxjnl-2015-207770.138}

Introduction Idiopathic pulmonary fibrosis (IPF) is a restrictive lung disease of unknown cause. The median survival time after diagnosis is $3-5$ years and there are limited treatments available. MUC5AC, MUC5B and TOLLIP in the $11 \mathrm{p} 15.5$ region have been shown to be associated with susceptibility to IPF. A variant in TOLLIP (rs5743890) has also been shown to be associated with both susceptibility and survival time however the effects were in opposite directions, i.e. the allele associated with increased susceptibility was also associated with increased survival time.

Methods We performed survival analysis on 612 European IPF cases passing QC using a Cox proportional hazards model adjusting for age, sex, first 10 principal components and study centre. 134 variants were genotyped in a $200 \mathrm{~kb}$ region on chromosome 11 covering MUC5AC, MUC5B and TOLLIP.

Results No SNPs in this region reached genome-wide significance $\left(\mathrm{p}<5 \times 10^{-8}\right)$. The most significant variant was rs56367042 (Hazard Ratio 3.38, 95\% CI [1.91, 5.96]; p = 2.74 $\times 10^{-5}$ ) which is located downstream of MUC5B. The next significant SNP was rs5743894 (Hazard Ratio 0.67, 95\% CI [0.54, 0.83 ]; $\mathrm{p}=2.24 \times 10^{-4}$ ) located in TOLLIP. For this SNP the allele we found associated with increased survival time has previously been reported as associated with increased susceptibility (showing a similar pattern to that reported for rs5743890) however this SNP has also been previously reported as not being associated with survival time. We found a proxy of rs5743890 $\left(\mathrm{R}^{2}=0.698\right)$ not to be associated with survival time ( $\mathrm{p}=$ $0.881)$.

Conclusions Our results did not replicate those previously reported; however they may support the hypothesis that variants in TOLLIP that increase susceptibility may also increase survival time. In future we will perform a GWAS for susceptibility and survival time and try and replicate the findings.

\section{P2 PLATELET REACTIVITY AS A POTENTIAL BIOMARKER IN IDIOPATHIC PULMONARY FIBROSIS}

MG Crooks, C Wright, S Fraser, AH Morice, SP Hart. Hull York Medical School, Hull, UK

10.1136/thoraxjnl-2015-207770.139

Introduction and objectives Heterogeneity of idiopathic pulmonary fibrosis (IPF) means it is difficult to identify those at highest risk of progression who are most likely to benefit from treatment. A biomarker that predicts disease activity, prognosis and treatment response would be beneficial. We previously reported increased platelet reactivity in $\mathrm{IPF}^{1}{ }^{1}$ and here we explore whether platelet reactivity warrants further investigation as a biomarker.
Methods Data were obtained from two studies: Study 1. a study of platelet reactivity in IPF; and Study 2. a pilot randomisedcontrolled trial of an investigational IPF treatment. Standard protocols were used to measure platelet-monocyte aggregate (PMA) formation, P-selectin expression, and fibrinogen binding in blood samples. Platelet reactivity in IPF was compared with controls. Correlation between platelet reactivity and forced vital capacity (FVC) was assessed. Study 2 data were used to assess the change in platelet reactivity in response to the intervention and correlation between baseline platelet reactivity, symptoms (KBILD) and exercise capacity (6MWD).

Results Study 1 included 13 IPF patients (mean \pm SD, Age 70.3 \pm 5.8 years, $69 \%$ male, FVC $91.9 \pm 17.8 \%$ predicted) and 12 controls (Age $66.2 \pm 10.2$, 66.7\% males). Study 2 included 19 IPF patients (Age $71.5 \pm 8.7,72 \%$ males, FVC $84.4 \pm 16.8 \%$ predicted). IPF patients demonstrated significantly increased platelet reactivity compared to controls $(\mathrm{P}<0.01)$. Platelet reactivity and FVC did not correlate. In study 2, stimulated platelets expressed significantly less P-selectin in response to the intervention $(\mathrm{P}=0.03)$. Unstimulated PMA formation moderately correlated with $\operatorname{KBILD}(\mathrm{r}=0.38 . \mathrm{P}=0.01)$, but other platelet markers showed no correlation with symptoms or exercise capacity.

Conclusion IPF patients exhibit increased platelet reactivity compared to controls. The reduction in platelet reactivity in response to an intervention may indicate responsiveness to treatment effect. Although there was no correlation between FVC and platelet activation, further investigation is warranted to assess associations between platelet reactivity and lung function decline and mortality.

\section{REFERENCE}

1 Crooks MG, Fahim A, Naseem KM, Morice AH, Hart SP. Increased platelet reactivity in idiopathic pulmonary fibrosis is medicated by a plasma factor. PLoS One 2014;9(10):e111347

\section{P3 PILOT STUDY TO TEST THE FEASIBILITY OF A PSYCHOLOGICAL SUPPORT WORKSHOP FOR PATIENTS NEWLY DIAGNOSED WITH IDIOPATHIC PULMONARY FIBROSIS (IPF) AND THEIR FAMILIES}

${ }^{1} \mathrm{~J}$ Cove, ${ }^{2} \mathrm{AM}$ Russell, ${ }^{1} \mathrm{~J}$ Wright, ${ }^{1} \mathrm{C}$ Hogben, ${ }^{1} \mathrm{M}$ Kokosi, ${ }^{1} \mathrm{~V}$ Mak, ${ }^{1} \mathrm{~F}$ Chua, ${ }^{2} \mathrm{~A}$ Wells, ${ }^{1} \mathrm{AM}$ Doyle, ${ }^{2} \mathrm{E}$ Renzoni. ${ }^{1}$ Royal Brompton and Harefield NHS Foundation Trust, London, UK; ${ }^{2}$ National Heart \& Lung Institute, Royal Brompton Hospital \& Imperial College, London, UK

\subsection{6/thoraxjnl-2015-207770.140}

Introduction IPF is associated with a poor prognosis, high symptom burden and limited treatment options. Psychological reactions are comparable to those seen in cancer patients, yet support services for IPF patients are less well developed. There is an urgent demand to develop Interstitial Lung Disease (ILD) services that better support the psychological needs of IPF populations. The aim of this pilot study is to characterise the psychological needs of both patients and carers and to test the feasibility of a workshop approach.

Methods Consultant ILD physicians identified patients recently diagnosed with IPF at our Unit, and referred them to the clinical nurse specialist. Patients were invited to attend a half day workshop with an accompanying 'guest'. The workshop, led by a clinical psychologist, included didactic teaching, interactive discussions and experiential learning. Topics covered coping with low mood and anxiety, symptom control and transitions towards the end of life. Four members of the clinical team were present 